\title{
New Splicing Variants of the Murine Damaged DNA Binding 2
}

\author{
Jun Hong Park ${ }^{1,2}$, YoonYi Nam ${ }^{3}$, Jungkee Kwon ${ }^{4}$ and Jun-Gyo Suh ${ }^{1,3 *}$ \\ ${ }^{1}$ Department of Medical Cenetics, College of Medicine, Hallym University, Chuncheon, Korea \\ ${ }^{2}$ Genetics of Development and Disease Branch, NIH/NIDDK, USA \\ ${ }^{3}$ Institute of Natural Medicine, Hallym University, Chuncheon, Korea \\ ${ }^{4}$ Biosafty Research Institute, College of Medicine, Chonbuk National University, Jeonju, Korea
}

\begin{abstract}
Damaged DNA binding (DDB) protein is an important gene in the repair of damaged DNA. DDB is a heterodimer (DDB1 and DDB2) protein, murine DDB2 has 10 exons about $1.5 \mathrm{~kb}$ in size (Genbank Accession No. AY027937). Here we identified five DDB2 variants (M1-M5) from various mouse tissues that are generated by alternative splicing. We used reverse transcription-PCR (RT-PCR) to identify splicing variants and isolated PCR products using an agarose-gel PCR purification kit. All isolated PCR products were cloned and the structure of splicing variants was confirmed by sequencing. The first splicing variant $M 1$ was generated by omission of exon 4 . The second splicing variant $M 2$, by omission of exons 4-5. The third variants M3 was generated by omission from the middle of exon 1 to exon 6 and was expressed in the heart. Fourth variants M4 was generated by omission of exon 2 and exons 4-7. M5, the last splicing variant was generated by omission of exons 4-7. M4 and M5 were expressed in the spleen. Analysis of tissue distribution by RT-PCR indicates that M1 is most highly expressed in the mouse brain. These results indicated that murine $D D B 2$ has five splicing variants and splicing variants expression patterns were different depending on mouse tissue. Further functional studies of each splicing variants will provide more information about the molecular mechanism of DDB2 function and DDB2 gene expression regulation.
\end{abstract}

Key words: DDB2, splicing variant, repair, mouse

(Received 26 February 2010; Revised version received 15 March 2010; Accepted 17 March 2010)

The damaged DNA binding (DDB) protein complex was identified as a heterodimeric complex that binds to the damaged DNA (Keeney et al., 1993). The DDB protein complex is composed of two subunits with molecular masses of 127 and $48 \mathrm{KDa}$ (known as DDB1 and DDB2), and the activity is dependent on the formation of the heterodimeric complex (Fujiwara et al., 1999). DDB strongly binds to damaged DNA, caused by UV light. The DDB binding to damaged DNA might suggest a direct role for DDB in nucleotide excision repair (NER) (Aboussekhra et al., 1995). Recognition of lesions for NER involves two mechanisms; transcription-coupled repair (TCR) and global genomic repair (GGR) (Bohret al., 1985). TCRs repair lesions on the transcribed strand of expressed genes, while GGRs repair lesions from

*Corresponding author: Jun-Gyo Suh, Department of Medical Genetics, College of Medicine, Hallym University, Chuncheon, Kangwon-do 200-702, Korea

Tel: $+82-33-248-2692$

Fax: +82-33-248-2696

E-mail: jgsuh@hallym.ac.kr both non-transcribed genomic DNA and the non-transcribed strand of expressed genes. DDB is thought to be involved in GGR UV-DDB protein recognizes the cyclobutane pyrimidine dimers (CPDs) at the initial step of global genomic repair. DDB2 also has a critical role for the initiation step of NER (Hwang et al., 1999). Moreover, the DDB1 has no mutation form, but DDB2 has several mutation forms in XP patients (Itoh et al., 1999).

Four human $D D B 2$ variants were identified from HeLa cells. These are generated by alternative splicing and, especially, some splicing variants are dominant negative inhibitors of DNA repair (Inoki et al., 2004). The splicing variants disrupted the complex formation between DDB1 and DDB2. Recent study shows that $D D B 2$ gene disruption leads to skin tumors and resistance to apoptosis after exposure to UV but not a chemical carcinogen (Itoh et al., 2004). The DDB2 gene structure and functional studies were very well studied but murine DDB2 splicing variants are still unknown.

In this study, we identified five splicing variants of $D D B 2$ from murine tissues. Murine DDB2 splicing variants were 


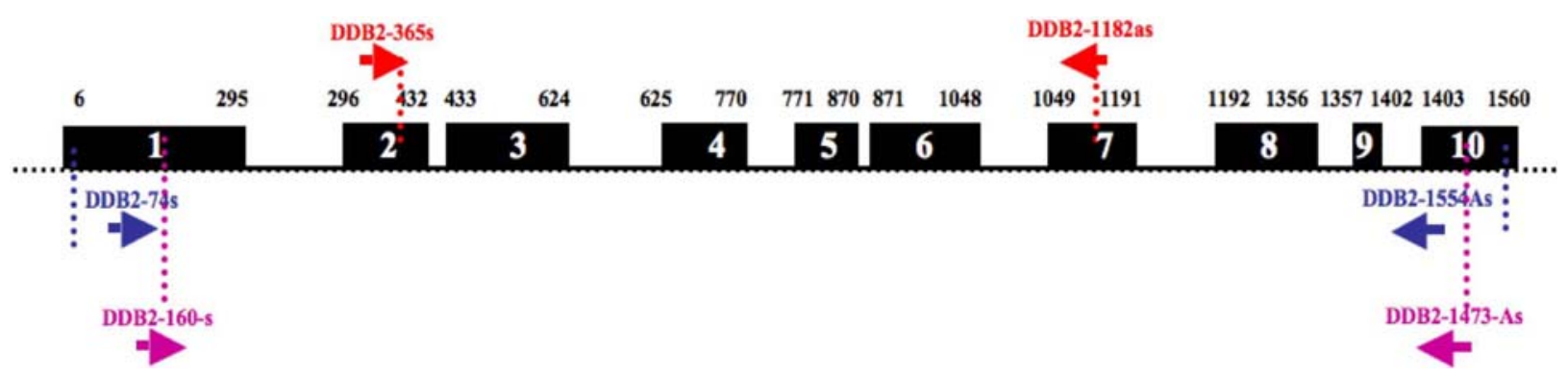

Figure 1. Mouse DDB2 genomic structure. Exons 1 to 10 are shown as black boxes. The numbers in the black boxes represent exon numbers and the above numbers are nucleotide numbers. Arrows indicated primers.

differently expressed depending on the murine tissue. These results indicated that murine DDB2 does not function as the same as human DDB2 variants and requires further study of DDB2 splicing variants function.

\section{Materials and Methods}

RNA preparation and reverse transcriptase polymerase chain reaction

Total RNA was extracted from various mouse tissues used Trizol according to the manufacturer's instructions (Invitrogen, Carlsbad, CA). The cDNAs were synthesized using the Reverse Transcription system (Promega, Madison, WI). Primary 3 software (Whitehead Institute, MIT Center for Genome Research, Cambridge, MA) was used to design specific primers for DDB2 and tubulin. Each foward and reverse primers were as followed. DDB2-74-s, 5'-atacgtagtcccttcctgttt-3'; DDB2-160-s, 5'-gagtacgtcatggctccc-3'; DDB2-365-s, 5'gtagcagcattgtcaggg-3'; DDB2-1264-As, 5'-gtgaggtgctgaaaatgg3'; DDB2-1473-As, 5'-ccatcttcatagtctttcatg-3'; DDB2-1554As, 5'-tactttggcctattaac-3'.

\section{cDNA cloning and Sequencing}

The amplified DNA fragments for wild-type and variants were separated on a $3 \%$ agarose gel and purified using Geneclean Turbo kit (Qbiogene, Carlsbad, CA). Cloning was done by the TOPO TA cloning kit (Invitrogen, Carlsbad, CA). Sequencing reactions were performed by Macrogene (Seoul, South Korea).

\section{Results and Discussion}

The mouse DDB2 gene has 10 exons and total size is about $1.5 \mathrm{kbp}$ (Figure 1). To determine the DDB2 gene expression pattern, first, we examined the distribution of $D D B 2$ gene expression in various mouse tissues (Figure 2A). The reverse transcriptase-PCR (RT-PCR) using DDB2-74-s and DDB2-1554-As, these primers amplified from exon 1 to exon

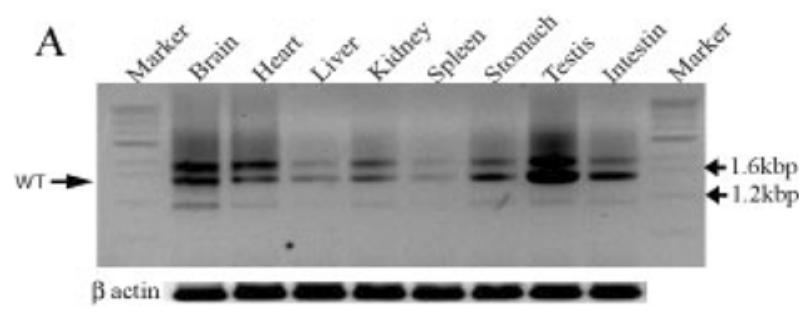

B

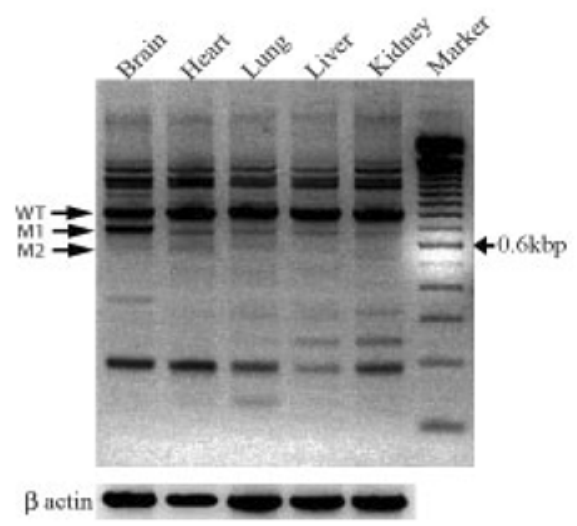

C

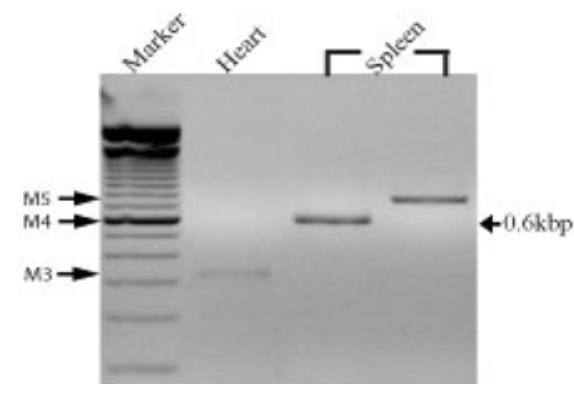

Figure 2. Mouse DDB2 expression and splicing variants in various mouse tissues. (A) DDB2 wild type (WT) distribution in various tissues by reverse-transcriptase PCR (RT-PCR). (B) DDB2 splicing variants (M1 and M2) in various tissues (C) Amplification of DDB2 splicing variants (M3, M4 and M5) cloned in TOPO vector in the heart and spleen. $\beta$-actin was used as an internal control for the PCR.

10. DDB2 wild-type gene was expressed more in the brain, heart and testes than other tissues. Recently studied, the human DDB2 splicing variants were more expressed in human brain and heart (Inoki, et al., 2004). We selected mouse

Lab. Anim. Res. | March, 2010 | Vol. 26, No. 1 
Wild type
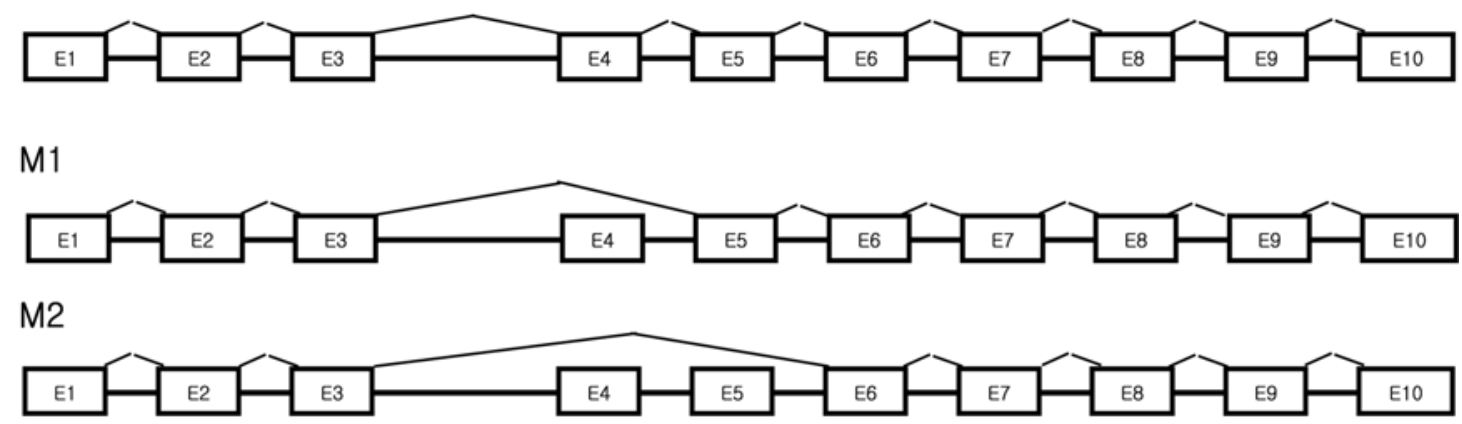

M3
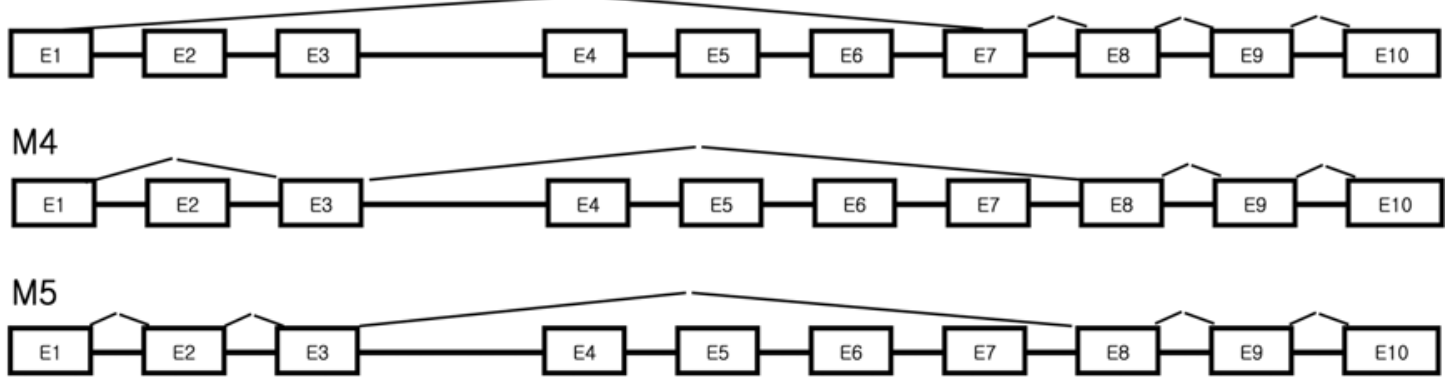

Figure 3. DDB2 wild type and variants gene structure. The structure of splicing variants was confirmed by sequencing. M1, omission of exon $4 ;$ M2, omission exons 4-5; M3, omission from the middle of exon 1 to exon 6 ; M4, omission of exon 2 and exons 4-7; M5, omission of exons 4-7.

brain, heart, lung, liver and kidney for detection of mouse DDB2 splicing variants, using primer DDB2-365-s and DDB21264 which amplified from exon 2 to exon 7 (Figure 2B). The amplified DNA fragments are separated on a $3 \%$ agarose gel, purified using agarose-gel purification kit. All fragments were cloned and sequenced. These DNA fragments identified a splicing variant product from the $D D B 2$ gene and other bands are non-specific band. The wild type DDB2 fragment was not omitted from any exons, and highly expressed in various tissues. The 146bp fragment was skipped in the splicing variant of $D D B 2, M 1$, which was generated by omission of exon 4 (Figure 2B). M2, the second splicing variant was generated by omission of exon 4 and 5 (Figure 2B). These two fragments were strongly expressed in the brain (Figure $2 \mathrm{~B})$. These results indicated that DDB2 gene was expressed in various mouse tissues being most highly expressed in the brain.

We investigated whether the other tissues has different splicing variants. Primers, DDB2-160-s and DDB2-1473-As, amplified the DDB2 gene from exon 1 to exon 10. The heart has one different variant, the splicing variant M3, generated by omission of the middle of exon 1 (starting point of amino acid sequence 179) and exons 2-6. The spleen has two splicing variants M4 and M5. M4 was generated by skipping exons 2 and 4-7. The last variant, M5, was generated by omitting from exon 4 to exon 7 (Figure 2C). These results indicated that the DDB2 gene has five different splicing variants and the splicing variants has different expression pattern in various mouse tissues (Figure 3).

Alternative splicing is a crucial mechanism for gene regulation and for generating proteomic diversity. Recent estimates indicate that the expression of nearly $95 \%$ of human multiexon genes involves alternative splicing (Roesser et al., 1993; Itohetal., 2006; Fedor, 2008). DDB2 is important for formation of DDB complex dimer and function (Stoyanova et al., 2009). The human DDB2 splicing variants type 2 is a dominant negative inhibitors of damaged DNA repair (Inoki, et al., 2004). But, numbers and functions of splicing variants of mouse DDB2 are still unknown. Our results showed that mouse DDB2 has five splicing variants and that each splicing variant expression pattern was different. We confirmed that mouse brain tissue has two splicing variants, the heart has one variant and the spleen has two variants. Alternative splicing contributes to genomic diversity and tissue specificity (Graveley, 2001). Comprehending tissue-specific alternative splicing requires an understanding of the regulatory network of protein-protein, protein-RNA and RNA-RNA interactions that are involved in this process (Graveley, 2009). Tissue-specific alternative splicing is thought to be controlled by differentially expressed splicing regulators and ubiquitously expressed splicing factors

Lab. Anim. Res. | March, 2010 | Vol. 26, No. 1 
of different concentrations and/or activity (Black, 2003). Moreover, splicing of individual pre-mRNA is frequently controlled by combinatorial or competitive effects of both activators and inhibitors (Mayeda et al., 1993). Tissue-specific alternative splicing events can be explained in part by tissuespecific expression of splicing factors, and the corresponding regulation of their target mRNA transcripts (David and Manley, 2008; Ohno et al., 2008). Further studies are required in order to define the splicing variants function and to regulation of wild type DDB2 expression in each tissues. In conclusion, we identified five mouse DDB2 splicing variants in various mouse tissues. Each tissue shows a different expression pattern. An exploration of DDB2 variants functional studies should provide new insights into the DDB2 gene mechanism and regulation.

\section{Acknowledgments}

This work was supported by Priority Research Centers Program through the National Research Foundation of Korea (NRF) funded by the Ministry of Education, Science and Technology (2009-0094074) and Hallym University.

\section{References}

Aboussekhra, A., Biggerstaff, M., Shivji, M.K., Vilpo, J.A., Moncollin, V., Podust, V.N., Protic, M., Hubscher, U., Egly, J.M. and Wood, R.D. (1995) Mammalian DNA nucleotide excision repair reconstituted with purified protein components. Cell, 80(6):859-868.

Black, D.L. (2003) Mechanisms of alternative pre-messenger RNA splicing. Annu. Rev. Biochem. 72:291-336.

Bohr, V.A., Smith, C.A., Okumoto, D.S. and Hanawalt, P.C. (1985) DNA repair in an active gene: removal of pyrimidine dimers from the DHFR gene of $\mathrm{CHO}$ cells is much more efficient than in the genome overall. Cell, 40(2):359-369.

David, C.J. and Manley, J.L. (2008) The search for alternative splicing regulators: new approaches offer a path to a splicing code. Genes Dev. 22(3):279-285.

Fedor, M.J. (2008) Alternative splicing minireview series: combinatorial control facilitates splicing regulation of gene expression and enhances genome diversity. J. Biol. Chem.
283(3):1209-1210

Fujiwara, Y., Masutani, C., Mizukoshi, T., Kondo, J., Hanaoka, F. and Iwai, S. (1999) Characterization of DNA recognition by the human UV-damaged DNA-binding protein. J. Biol. Chem. 274(28):20027-20033.

Graveley, B.R. (2001) Alternative splicing: increasing diversity in the proteomic world. Trends Genet. 17(2): 100-107.

Graveley, B.R. (2009) Alternative splicing: regulation without regulators. Nat. Struct. Mol. Biol. 16(1):13-15.

Hwang, B.J., Ford, J.M., Hanawalt, P.C. and Chu, G. (1999) Expression of the p48 xeroderma pigmentosum gene is p53dependent and is involved in global genomic repair. Proc. Natl. Acad. Sci. USA 96(2): 424-428.

Inoki, T., Yamagami, S., Inoki, Y., Tsuru, T., Hamamoto, T., Kagawa, Y., Mori, T. and Endo, H. (2004) Human DDB2 splicing variants are dominant negative inhibitors of UVdamaged DNA repair. Biochem. Biophys. Res. Commun. 314(4):1036-1043.

Itoh, M., Nagatomo, K., Kubo, Y. and Saitoh, O. (2006) Alternative splicing of RGS8 gene changes the binding property to the M1 muscarinic receptor to confer receptor type-specific Gq regulation. J. Neurochem. 99(6):1505-1516.

Itoh, T., Cado, D., Kamide, R. and Linn, S. (2004) DDB2 gene disruption leads to skin tumors and resistance to apoptosis after exposure to ultraviolet light but not a chemical carcinogen. Proc. Natl. Acad. Sci. USA 101(7):2052-2057.

Itoh, T., Mori, T., Ohkubo, H. and Yamaizumi, M. (1999) A newly identified patient with clinical xeroderma pigmentosum phenotype has a non-sense mutation in the DDB2 gene and incomplete repair in (6-4) photoproducts. J. Invest. Dermatol. 113(2):251-257.

Keeney, S., Chang, G.J. and Linn, S. (1993) Characterization of a human DNA damage binding protein implicated in xeroderma pigmentosum E. J. Biol. Chem. 268(28):2129321300.

Mayeda, A., Helfman, D.M. and Krainer, A.R. (1993) Modulation of exon skipping and inclusion by heterogeneous nuclear ribonucleoprotein A1 and pre-mRNA splicing factor SF2/ASF. Mol. Cell Biol. 13(5):2993-3001.

Ohno, G., Hagiwara, M. and Kuroyanagi, H. (2008) STAR family RNA-binding protein ASD-2 regulates developmental switching of mutually exclusive alternative splicing in vivo. Genes Dev. 22(3):360-374.

Roesser, J.R., Liittschwager. K. and Leff, S.E. (1993) Regulation of tissue-specific splicing of the calcitonin/calcitonin gene-related peptide gene by RNA-binding proteins. J. Biol. Chem. 268(11): 8366-8375.

Stoyanova, T., Roy, N., Kopanja, D., Raychaudhuri, P. and Bagchi, S. (2009) DDB2 (Damaged-DNA binding protein 2) in nucleotide excision repair and DNA damage response. Cell Cycle, 8(24):4067-4071. 